\title{
Poster: Excess Propagation Loss for Train-to-X Communications in Curved Subway Tunnels
}

\author{
Ke Guan , Bo Ai , Zhangdui Zhong , Carlos F. López, Lei Zhang , and Cesar Briso-Rodríguez \\ State Key Laboratory of Rail Traffic Control and Safety, Beijing Jiaotong University, 100044, Beijing, China \\ ETSI y Sistemas de Telecomunicación, Universidad Politécnica de Madrid, 28031, Madrid, Spain \\ E-mail: ke.guan.bjtu@qq.com
}

\begin{abstract}
Wave propagation experiences the excess loss in curved tunnels, which is highly desired to involve in the network planning for train-to-x communications. Extensive propagation measurements are conducted in a curved subway tunnel. By subtracting the measured received power in the curved tunnel from the simulated reference power in the equivalent straight tunnel, the excess loss resulting from the tunnel curve is obtained. Finally, tables and figures are provided to quantitatively reveal how the radius of curvature and frequency influence the excess loss in subway tunnels.
\end{abstract}

\section{INTRODUCTION}

No matter for public or dedicated train-to-x communication systems, a full understanding of the propagation is mandatary for their deployments in subway tunnels. During the last decades, many works related to the propagation in tunnels have been published and attracted intensive attention [1], [2]. However, most of these models are sophisticated for the straight tunnels. The excess loss of tunnel curves in different subway tunnel conditions and system configurations has been rarely investigated before. The main contribution of this paper is to present the first-hand measurement results in the subway tunnels to quantitatively reflect how the radius of curvature and frequency influence the excess loss in subway tunnels.

\section{Measurement Campaign}

\section{A. Test system}

The test system is introduced as follows:

1) Transmitting system: the transmitting system is comprised of a continuous wave (CW) transmitter (Tx) and a panel antenna. Since totaly three distinguished frequencies of interest are measured, three transmitters and corresponding antennas are utilized. For the measurements at $920 \mathrm{MHz}$, a Tx with $27 \mathrm{dBm}$ output power, and an antenna working at the frequency band 902-928 $\mathrm{MHz}$, with $8 \mathrm{dBi}$ gain, $75^{\circ}$ horizontal and $65^{\circ}$ vertical beam widths are used. In the measurements at 2400

This work is supported by the NNSF of China under Grant U1334202, 863 project under Grant 2014AA01A706, the Fundamental Research Funds for the Central Universities (No. 2014RC018), and State Key Lab of Rail Traffic Control and Safety Project under Grant RCS2014ZT32, and being developed under the framework of INNPACTO TECRAIL research project IPT-2011-1034-37000 funded by the Spanish Ministry of Economy and Competitiveness.
$\mathrm{MHz}$, a Tx with $24 \mathrm{dBm}$ output power is employed to be the Tx; an antenna working at the frequency band $2400-2500 \mathrm{MHz}$, with $14 \mathrm{dBi}$ gain, $30^{\circ}$ horizontal and $30^{\circ}$ vertical beam widths is utilized. In the cases of $5705 \mathrm{MHz}$, the Tx is a transmitter with $22 \mathrm{dBm}$ output power; the antenna works at the frequency band 5470$5850 \mathrm{MHz}$, with $19 \mathrm{dBi}$ gain, $16^{\circ}$ horizontal and $16^{\circ}$ vertical beam widths. In order to avoid possible damage in the measurements, the transmitting system is attached to a pylon located on land.

2) Receiving system: it is composed of a broadband antenna, an amplifier, and a spectrum analyzer, located on a standard subway train. The receiving antenna is with $8.5 \mathrm{dBi}$ gain, $85^{\circ}$ horizontal and $70^{\circ}$ vertical beam widths, located in the front car of the train. An amplifier working at the frequency band $500-6000 \mathrm{MHz}$ is used to amplify the received power. $\mathrm{A}$ spectrum analyzer is employed to send the captured received signal power to a laptop, stored for the later processing.

\section{B. Measurement environment}

The measurements are conducted in a curved arched tunnel of Madrid's subway. As shown in Fig. 1, the tunnel includes arched walls and roof, but a plane floor, more like a semicircle. Positions and configurations of the transmitting and receiving systems along the train route inside the tunnel with dimensions $8.41 \mathrm{~m} \times 6.87 \mathrm{~m}$ are illustrated in Fig. 1 as well.

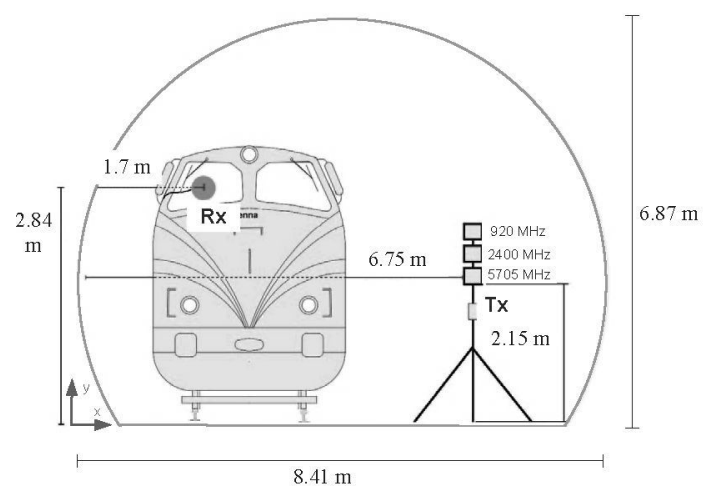

Fig. 1. Positions of Tx and Rx in the measured tunnel

In the measurements, the three carrier frequencies mentioned above are tested in the two durations with radii of 
curvatures with horizontal polarized (H-pol.) antennas to get the influence of frequency and curve, respectively.

\section{EXCESS LOSS OF TUNNEL CURVE}

In order to extract the excess loss of tunnel curve from the total power, a ray tracing simulator (verified in both straight and curved tunnels) is employed to simulate the reference received power by assuming the curved tunnel to be straight. By subtracting the measured power in the curved tunnel from the simulated reference power, the excess loss resulting from the tunnel curve (with the unit of $[\mathrm{dB} / 100 \mathrm{~m}]$ ) is obtained. In the implementation, such as the network planning, the excess loss can be directly added to the propagation loss in the straight tunnel to predict the total propagation loss inside curved tunnels. Fig. 2 illustrates the excess losses resulting from the tunnel curve with mean values 3.8-6.7 dB/100 m extracted from extensive repeated measurements.

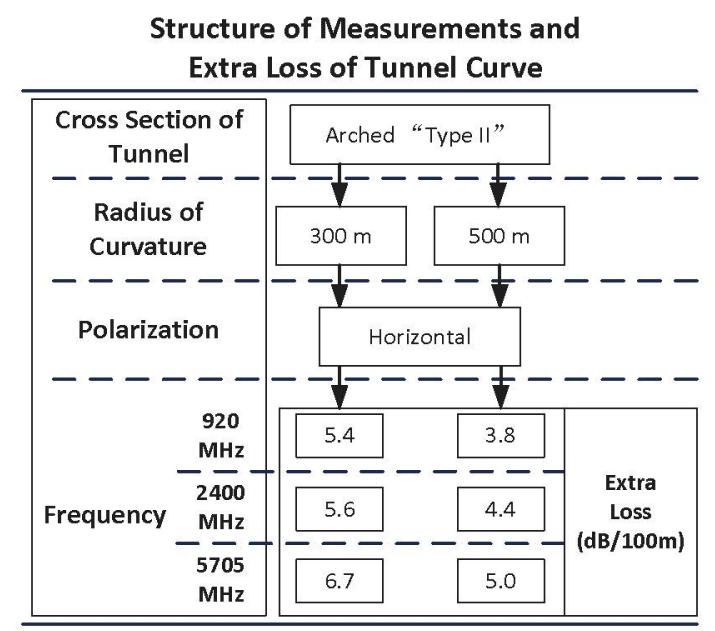

Fig. 2. Excess loss of tunnel curve in the measurements

\section{ANALYSIS OF THE EXCESS LOSS OF TUNNEL CURVE}

In this section, how the radius of curvature and frequency influence the excess loss is quantitatively analyzed based on the values (see Fig. 2) extracted from the measurements.

\section{A. Excess loss and radius of curvature}

Fig. 3 shows the excess loss versus radius of curvature in the tunnel at various frequencies with horizontal polarization. It can be found that a smaller radius of curvature corresponds to a higher excess loss at all the three frequencies. This observation is in line with common sense.

\section{B. Excess loss and frequency}

Fig. 4 illustrates the excess loss versus frequency in the tunnel with horizontal polarization. From this figure, it can be noticed that the higher frequency results in a higher excess loss if the other factors do not change. This finding implies that the relatively lower attenuation rate of higher frequencies in the straight tunnels is nullified by the excess loss of tunnel

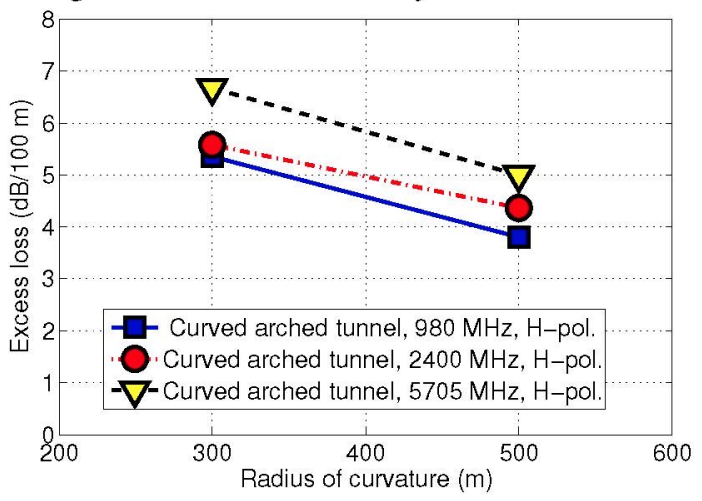

Fig. 3. Excess loss versus radius of curvature in the tunnel at various frequencies with horizontal polarization

curve. Thus, in the curved tunnel, the total attenuation rates of various frequencies tend to be similar. In cases of sharp curves, the total attenuation even could increase with the increase of frequency in curved tunnels, which is contradictory to the cases in straight tunnels dominated by waveguide effect. Thus, which frequency has the relatively lower attenuation rate depends on the specific conditions, such as the radius of the curvature, frequency, etc.

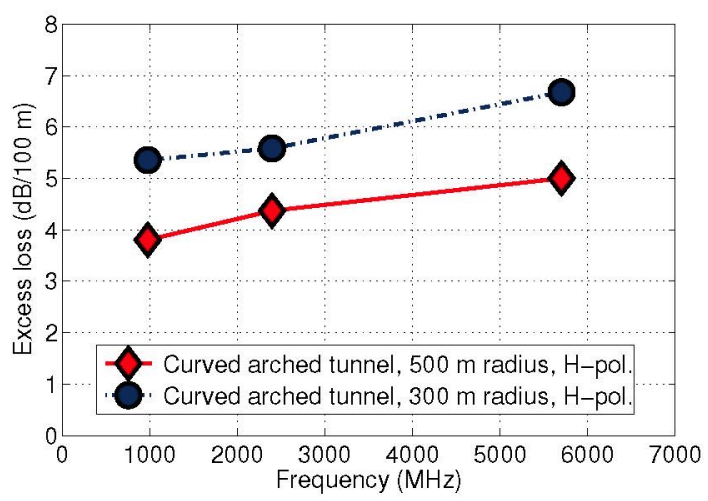

Fig. 4. Excess loss versus frequency in the tunnel with horizontal polarization

\section{CONCLUSION}

This paper presents the excess loss of the tunnel curve based on measurements in a real subway tunnel at $920 \mathrm{MHz}, 2400$ $\mathrm{MHz}$, and $5705 \mathrm{MHz}$. 3.8-6.7 dB/100 $\mathrm{m}$ excess losses are obtained under various conditions. It is found that a smaller radius of curvature leads to a higher excess loss. Moreover, the higher frequency results in a higher excess loss, which means that the relatively lower attenuation rate of higher frequencies in the straight tunnels is reduced by the tunnel curve.

\section{REFERENCES}

[1] A. Hrovat, G. Kandus, and T. Javornik, "A survey of radio propagation modeling for tunnels," IEEE Communications Surveys \& Tutorials, vol. 16, no. 2, pp. 658-669, Second Quarter 2014.

[2] K. Guan, Z. Zhong, J. I, Alonso, and C. Briso, "Measurement of distributed antenna systems at $2.4 \mathrm{GHz}$ in a realistic subway tunnel environment," IEEE Transactions on Vehicular Technology, vol. 61, no. 2, pp. 834-837, Feb. 2012. 